\title{
Dual anisotropicity comprising 3D printed structures and magnetic nanoparticle assemblies: towards the promotion of mesenchymal stem cell osteogenic differentiation
}

\author{
Ke Hu ${ }^{1,2}$, Tingting $Y u^{3}$, Shijia Tang ${ }^{1}$, Xueqin X $u^{2}$, Zhaobin Guo ${ }^{4}$, Jun Qian', Yi Cheng ${ }^{1}$, Yinyi Zhao ${ }^{2}$, Sen Yan ${ }^{5}$, \\ Huijie Zhang ${ }^{2}$, Mengqi Wan², Chunyue Du², Yiwei Feng ${ }^{2}$, Qi Liu², Zhuxiao Gu', Bo Chen ${ }^{6}$, Feimin Zhang $\mathbb{D}^{1,2}$ and \\ Ning Gu $\mathbb{1}^{2,5}$
}

\begin{abstract}
Leveraging physical factors in cellular microenvironments to promote adipose tissue-derived stem cell (ADSC) osteogenic differentiation has emerged as a new strategy in the development of scaffolds for bone tissue engineering. Anisotropicity is one of those factors of interest; however, the utilization of anisotropicity to promote ADSC osteogenic differentiation is still not efficient. In this study, we designed a substrate with a dual anisotropic structure fabricated via a combination of 3D printing and magnetic field-induced magnetic nanoparticle assembly techniques. These dual anisotropic structures have a scale hierarchy, and the scale of the magnetic nanoparticle assemblies matches that of a single ADSC. This is in contrast to conventional anisotropic osteogenic induction scaffolds that have anisotropic structures at only one scale and at an order of magnitude different from single ADSCs. ADSCs cultured on substrates with such structures have significantly higher osteogenic marker expression, e.g., ALP, at both the protein and mRNA levels, and more calcium nodule formation was also found, suggesting a stronger tendency toward osteogenic differentiation of ADSCs. RNA-seq data revealed that alterations in kinase signaling pathway transduction, cell adhesion, and cytoskeletal reconstruction may account for the elevated osteogenic induction capacity. These data support our hypothesis that such a structure could maximize the anisotropicity that ADSCs can sense and therefore promote ADSC osteogenic differentiation.
\end{abstract}

\section{Introduction}

Bone tissue engineering (BTE) has emerged as a promising strategy to repair bone defects mainly because of its advantages, such as relatively limitless supplementation and a low risk of disease transmission ${ }^{1}$, compared to

\footnotetext{
Correspondence: Feimin Zhang (fmzhang@njmu.edu.cn) or

Ning Gu (guning@seu.edu.cn)

'Jiangsu Key Laboratory of Oral Diseases, Affiliated Hospital of Stomatology, Nanjing Medical University, Nanjing, Jiangsu, China

${ }^{2}$ Laboratory of Oral Regenerative Medicine Technology, School of Biomedical Engineering and Informatics, Department of Biomedical Engineering, Nanjing

Medical University, Nanjing, Jiangsu, China

Full list of author information is available at the end of the article

These authors contributed equally: Ke Hu, Tingting Yu
}

conventional bone graft approaches. However, some hurdles still limit the clinical application of BTE. One of those hurdles is controlling the directional osteogenesis differentiation of stem cells seeded in the scaffold, which is still inadequate. Conventional approaches involve using substantial amounts of osteoinductive growth factors or other chemicals, which lack efficiency for osteogenesis induction and might raise safety concerns in clinical settings ${ }^{1,2}$. Researchers recently found that physical cues in the microenvironment can control stem cell fate ${ }^{3}$ and that particular setting can induce the osteogenesis of stem cells $^{4}$. For example, Young's modulus ${ }^{5}$, stress relaxation ${ }^{6}$, and the topography ${ }^{7-9}$ of the extracellular matrix have

\section{(c) The Author(s) 2021}

(c) (i) Open Access This article is licensed under a Creative Commons Attribution 4.0 International License, which permits use, sharing, adaptation, distribution and reproduction cc. in any medium or format, as long as you give appropriate credit to the original author(s) and the source, provide a link to the Creative Commons license, and indicate if changes were made. The images or other third party material in this article are included in the article's Creative Commons license, unless indicated otherwise in a credit line to the material. If material is not included in the article's Creative Commons license and your intended use is not permitted by statutory regulation or exceeds the permitted use, you will need to obtain permission directly from the copyright holder. To view a copy of this license, visit http://creativecommons.org/licenses/by/4.0/. 
been reported to be key physical factors in determining the fates of different types of stem cells. Leveraging these physical factors in designing artificial extracellular matrices that can direct stem cell differentiation with high efficiency while lowering the doses of osteoinductive growth factors can both facilitate BTE clinical application and shed light on the mechanism of stem cell osteogenesis.

Anisotropic topographic structures are ubiquitous in natural ECM at different scale levels (tissue ${ }^{10}$, cellular ${ }^{11}$, subcellular $^{12}$, etc.). Previous studies demonstrated that substrates with anisotropic structures have higher induction capabilities in the osteogenic differentiation of stem cells ${ }^{13-15}$. However, most studies have focused on investigating anisotropic structures on a scale that is an order of magnitude higher or lower than that of single cells. Although anisotropicity at such scale levels is also present in real tissue or cells and might regulate activities primarily at the corresponding scales (the tissue level or subcellular level), from the perspective of single cells, the microenvironment they reside is still relatively isotropic. More importantly, studies of the hierarchy of anisotropicity, in other words, the combination of anisotropic structures at both the tissue and cell levels, in the osteogenic differentiation of stem cells were missing in most studies. However, this hierarchy is normally present in living tissue and therefore inspired us to investigate the osteogenesis induction capacity of such hierarchical anisotropicity ${ }^{16}$.

With this idea in mind, we leveraged state-of-the-art "top-down" 3D printing technology in combination with the "bottom-up" technique we previously developed, namely, "field-assisted magnetic nanoparticle self-assembly" technology ${ }^{17-19}$, to fabricate a substrate with an anisotropic structure on both the hundred-micron scale and $\sim 10$-micron scale. This dual anisotropic structure has a scale hierarchy, and the scale of the magnetic nanoparticle assemblies approaches that of a single ADSC. Alizarin red staining, q-PCR, western blot, and RNA-seq data consistently demonstrated that ADSCs cultured on substrates with this type of structure have a stronger osteogenesis tendency. In particular, RNA-seq data revealed that genes that mainly responded to dual anisotropic structures were enriched in cell adhesion, cytoskeleton, and kinase signaling pathways, including MAPK pathways and PI3K-Akt pathways. These data support our hypothesis that such a structure could maximize the microenvironmental anisotropicity sensed by ADSCs and therefore validate the feasibility of this strategy for promoting ADSC osteogenic differentiation.

\section{Results and discussion}

We endeavored to fabricate a platform that has anisotropic structures with scales of different orders of magnitude and explored which anisotropic parameters endowed the platform with the highest osteogenic differentiation induction capabilities. Specifically, a 3D printing technique was employed to fabricate PDMS stripes with both widths and distances between two stripes on the hundreds of micrometers scale, while a (magnetic) field-assisted approach was employed to fabricate magnetic nanoparticle assemblies (MNAs) on top of the PDMS stripes on the ten-micrometer scale (Fig. 1).

We first explored the effects of substrates with anisotropic structures on ADSC osteogenic differentiation at the hundreds of micrometers scale. 3D printing was used to fabricate PDMS features with different sizes and anisotropicity on a PDMS-coated glass slide. By adjusting the size of the injection head and pressure, the width of the PDMS stripes and the gap widths between stripes, and ultimately the anisotropicity of the structure (L/W ratio), can be precisely controlled. We fabricated a series of substrates with various anisotropicities (Fig. S1) and compared the behaviors of ADSCs cultured on these substrates. Observation by confocal microscopy revealed that the cells exhibited an elongated morphology with a long axis well aligned with the direction of the PDMS stripes when cultured on highly anisotropic stripes. This is in contrast to those cultured on PDMS substrates with low anisotropicity, where the majority of the cells exhibited a typical flask-cultured, polygonal-like morphology (Fig. S2). In addition, an orthogonal experiment was performed to explore the expression of several osteoblast marker genes in ADSCs cultured on substrates with stripe widths of $100 \mu \mathrm{m}, 210 \mu \mathrm{m}$, and $410 \mu \mathrm{m}$, as well as spaces between stripes of $50 \mu \mathrm{m}, 100 \mu \mathrm{m}, 200 \mu \mathrm{m}$, and $300 \mu \mathrm{m}$ (Fig. S3). In most cases, ADSCs cultured on stripes with widths of $210 \mu \mathrm{m}$ had the highest osteogenic markers expression, regardless of the spaces between stripes, while in the $210 \mu \mathrm{m}$ group, ADSCs cultured on $200 \mu \mathrm{m}$ spaces between stripes had the highest expression of 5 osteogenic genes among a total of 6 measured genes. Based on these results, a substrate with a $210 \mu \mathrm{m}$ stripe width and a $200 \mu \mathrm{m}$ space between stripes with optimal osteogenic differentiation induction efficiency was used in the following studies.

Next, we fabricated anisotropic structures on the micrometer scale on a PDMS substrate with the magnetic nanoparticle assembly technique we developed previously ${ }^{17,20}$. In this work, ferumoxytol, the latest inorganic nanodrug approved by the FDA, which is composed of an iron oxide core ( $7 \mathrm{~nm}$ in diameter) and a dextran (PSC) shell (20 nm in thickness), was chosen as the building block. Specifically, ferumoxytol magnetic nanoparticles $\left(\gamma-\mathrm{Fe}_{2} \mathrm{O}_{3} @ \mathrm{PSC}\right)$ were first mixed with poly-L-lysine (PLL). Figure S4 demonstrates the transmission electron microscopy (TEM) and hydrodynamic size of $\gamma$ $\mathrm{Fe}_{2} \mathrm{O}_{3} @ \mathrm{PSC}$ magnetic nanoparticles before and after 

A. Printing of PDMS precursor
B. Heat solidification
C. Field induced assembly
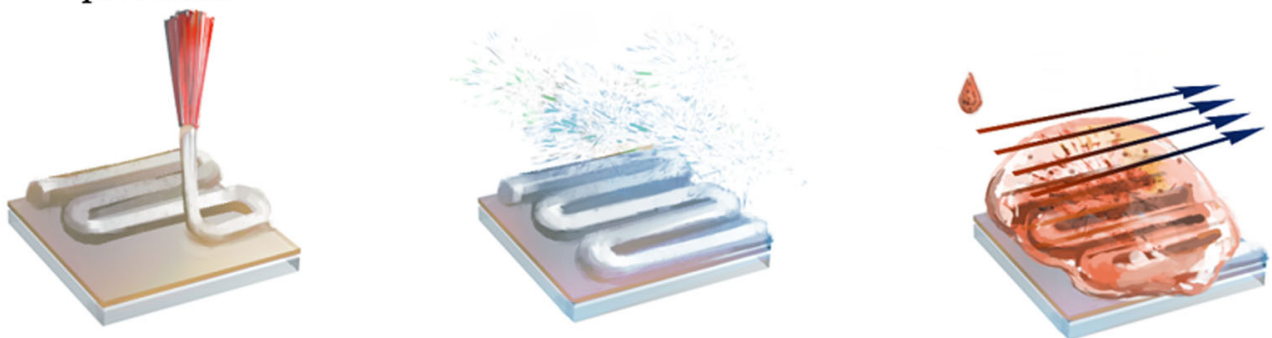

D. Assemblies deposition by evaporation
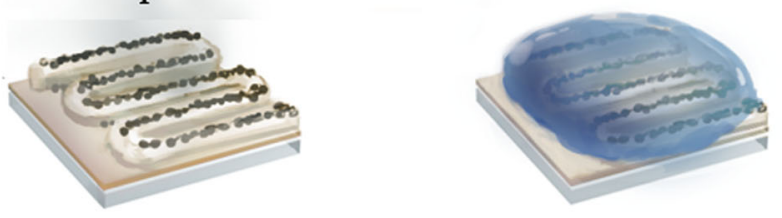

E. Collagen coating

PDMS

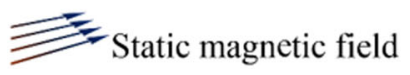

Magnetic nanoparticle

\section{Collagen Type I Rat Tail+Dopamine}

Fig. 1 Schematic illustration of the fabrication procedures for PDMS substrates with dual anisotropic structures via the combination of 3D printing (top-down) and field-induced self-assembly of magnetic nanoparticles (bottom-up). A Printing of PDMS precursors. B Heat solidification. C Field induced assembly of magnetic nanoparticles (MNPs). D Assemlies deposition by evaporation. E Collagen coating.

mixing with PLL. While negligible morphological changes of single particles were observed after mixing, both TEM and hydrodynamic size demonstrated a moderate aggregation of nanoparticles after coating with PLL. This was expected since such a coating could significantly change the surface charge of the nanoparticles and thus change the equilibrium status of the nanoparticles during the coating process. In fact, such aggregation could increase the responsiveness of particles to a magnetic field and therefore facilitate the field-induced assembly of particles. Next, these PLL-coated particles were assembled along the long axis of the $3 \mathrm{D}$ printed structure on a PDMS substrate with or without the presence of a static magnetic field (SMF) (Fig. 1). When SMF was absent, particles were assembled at the bottom of the PDMS stripes driven by the surface structure of the $3 \mathrm{D}$ printed PDMS substrate, therefore resulting in a direction of assembly that aligned with those stripes. However, when SMF was present, the magnetic nanoparticles formed anisotropic assemblies in the solution, and these assemblies might sediment before the solvent completely evaporated (Fig. 2K). Energy dispersive spectrometry (EDS) results based on scanning electronic microscopy (SEM) images showed that the magnetic nanoparticles formed oriented assemblies along the bottom of the PDMS stripes when SMF was absent; in contrast, oriented assemblies formed on the top of stripes when SMF was present (Fig. 2). Stronger anisotropic characteristics were observed in the magnetic PDMS composite prepared under a static magnetic field, as indicated by the height of the peak in the FMR spectrum (Fig. 2G, H, I). We have previously found that these phenomena are directly related to the field-induced assembly process during solvent evaporation. To increase the adhesion of ADSCs to MNA, collagen was coated on MNA and covalently stabilized with dopamine.

With this method, we fabricated three groups of substrates, namely, MNP- (control), with the only 3D printed PDMS stripes, and MNP + MNA - and MNP+MNA+, with magnetic nanoparticles assembled on top of printed substrates in the absence or presence of SMF. The macroscopic and optical microscope images and elemental analysis of Group MNP- (A, D, G), Group MNP+MNA$(\mathrm{B}, \mathrm{E}, \mathrm{H})$, and Group $\mathrm{MNP}+\mathrm{MNA}+(\mathrm{C}, \mathrm{F}, \mathrm{I})$ are displayed in Fig. 2.

To investigate the osteogenesis induction efficiency of these substrates, SD rat adipose-derived mesenchymal stem cells were inoculated into three groups. Since the 


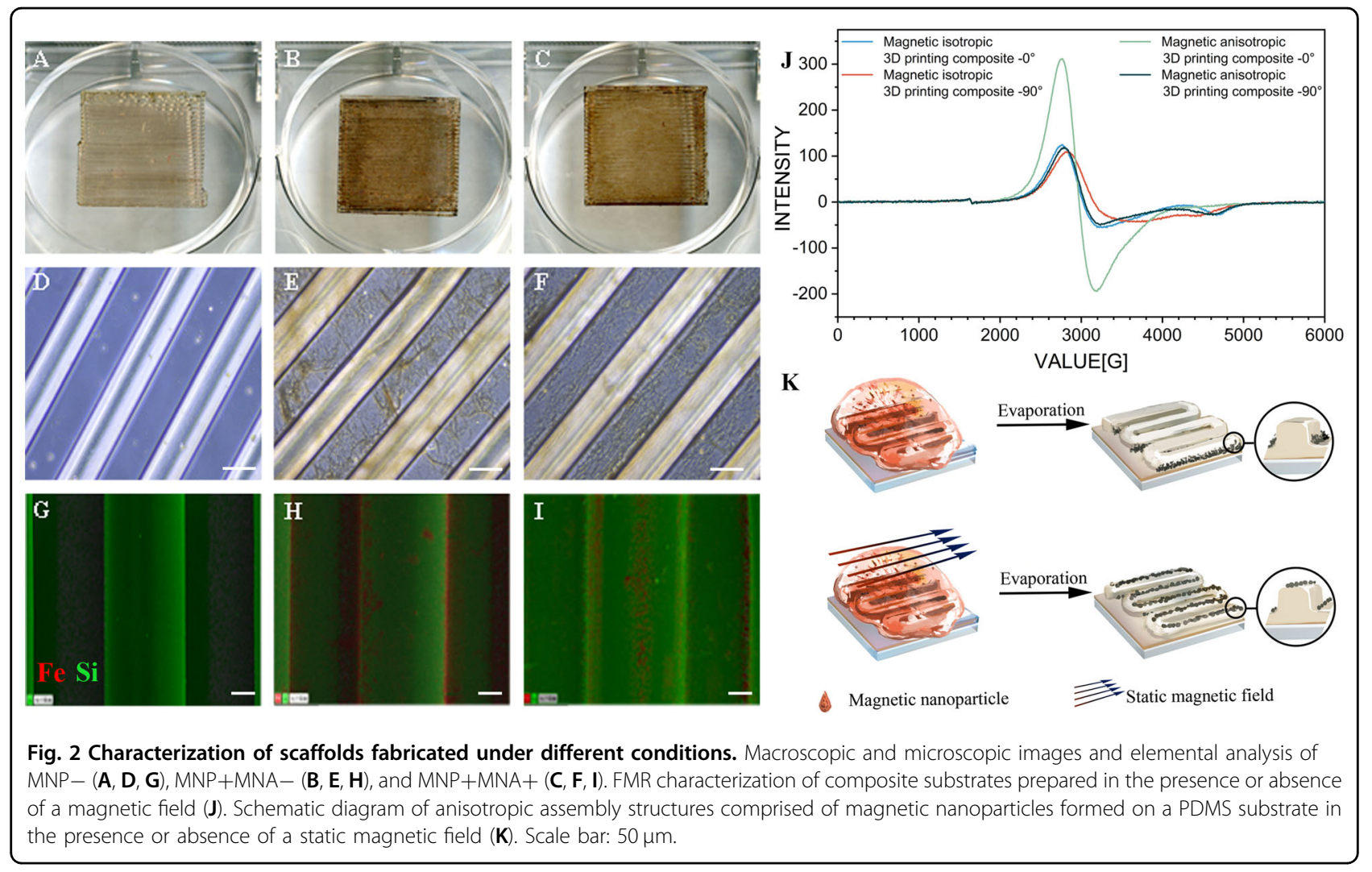

transparency of the substrates was compromised by the brownish nanoparticles, which hindered the observation of cells seeded on the substrate with a phase-contrast microscope, the F-actin-stained cells were observed with a confocal microscope 3 days after seeding (Fig. 3). Higher cell densities were observed in groups with magnetic nanoparticles (B and $\mathrm{C}$ ) compared to the control group, and both the morphology of cells and the actin filaments within cells were found to be well aligned with the direction of the PDMS stripes and nanoparticle assemblies. It is worth mentioning here that we focused on the top plane of stripes and imaged only those cells that were sitting on top of the stripes with a confocal microscope. With this method, we eliminated cells in the spaces between the stripes, where we assumed that the cells were not fully guided by the anisotropic structures.

We detected the expression of the osteogenesis marker ALP with an ALP activity assay kit at 4 and 7 days after seeding. An obvious increase was observed in Group $\mathrm{MNP}+\mathrm{MNA}$ - and Group MNP+MNA + compared with Group MNP- on day 4, and significantly higher expression of ALP was found in Group MNP+MNA+ on day 7. Similarly, there were substantially more calcium nodules in Group MNP+MNA+ than in the other 2 groups according to the Alizarin Red staining assay performed on day 14. Of note, we also found that the PDMS stripe with a width of $600 \mu \mathrm{m}$ (Fig. S5) without MNPs had a minor effect on inducing osteogenesis of ADCSs, as evidenced by Alizarin staining. Considering the size of the stripes, which substantially exceeded the size of single cells, cells cultured on the stripes were in an isotropic environment, which was substantiated by the negligible alignment of the actin cytoskeleton along with the stripes (Fig. S5A and B). These results show that the osteogenesis of ADSCs is the direct result of anisotropic structures (Fig. 3 and Fig. S5C).

To further validate these results, we measured the expression of several key osteogenesis markers at the mRNA level with RT-PCR (Fig. 4A) and at the protein level with Western blotting (Fig. 4B). We found that the expression of ALP in Group MNP+MNA+ was significantly higher than that in Group MNP- ( 2.4-fold $)$ and Group MNP+MNA - ( 1.5-fold) after 14 days of cell culture. Approximately 1.8 -fold higher relative expression of BMP2 was measured in Group MNP+MNA+ than in the other two groups. The expression of RUNX2 increased approximately 1.6 -fold compared with that of Group MNP-fold and 2.3-fold compared with that of Group MNP+MNA-. The Western blot results were consistent with the PCR data. These sets of data further support our hypothesis that substrates with anisotropicity at dual scale levels (printed structures + assembled magnetic nanoparticles) can collaboratively induce the osteogenic differentiation of ADSCs. 

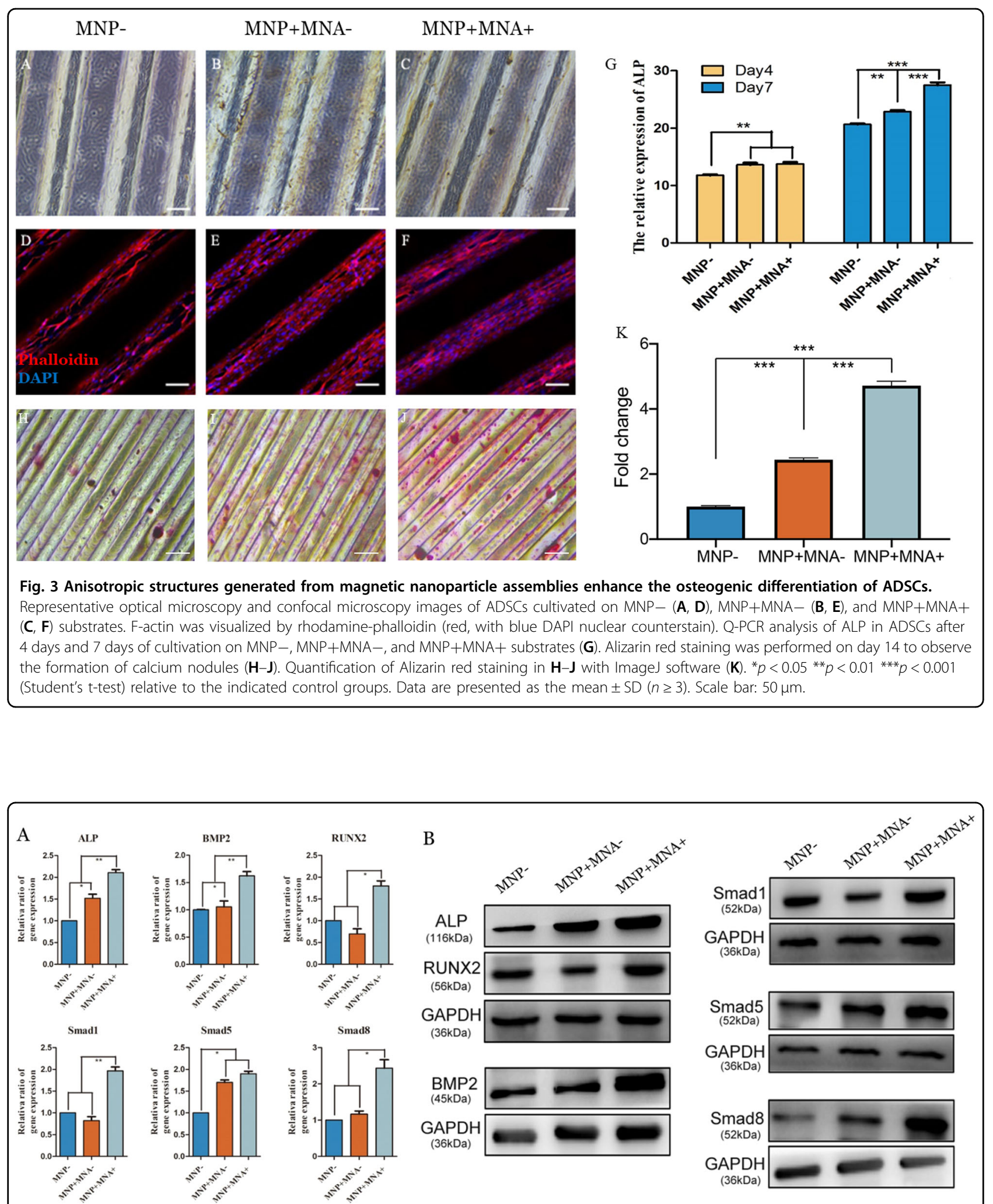

Fig. 4 Anisotropic structures generated from magnetic nanoparticle assemblies upregulate osteogenesis signaling pathways. $Q P C R$ analysis of ALP, BMP2, RUNX2, and Smad1/5/8 in ADSCs after 14 days of cultivation on MNP-, MNP+MNA-, and MNP+MNA+ substrates. Statistical significance is shown compared to Group MNP- (control). ${ }^{*} p<0.05{ }^{* *} p<0.01$ (Student's t-test). Data are presented as the mean \pm SD $(n \geq 3)(\mathbf{A})$. Western blot analysis of ALP, BMP2, RUNX2, and Smad1/5/8 in ADSCs cultivated on MNP-, MNP+MNA-, and MNP+MNA+ substrates (B). 
To delineate the key genes and pathways that account for the elevated osteogenesis induction by anisotropicity generated from magnetic nanoparticle assemblies (MNAs), we undertook an RNA-seq-based transcriptomic approach for gene expression comparison across the 3 groups. Based on the 3 biological replicates, the RNA-seq assay detected 106 and 213 differentially expressed genes (DEGs) compared to the MNP - control group in the $\mathrm{MNP}+\mathrm{MNA}-$ and $\mathrm{MNP}+\mathrm{MNA}+$ groups, respectively, with cutoff values of $p<0.05$ and $\left|\log _{2} \mathrm{FC}\right|>1$ (Fig. S6). Next, we performed a further comparison of the three groups in pairs. By categorizing the DEGs into defined cellular pathways by performing KEGG pathway analysis, we found that metabolic pathways, phagosomes, and the Ras signaling pathway were the majority of the pathways mapped across the 3 groups, which was consistent with the involvement of these pathways in osteogenesis. In addition to demonstrating the overlapping distribution of the above signaling pathways, the dot plot map also shows the proportions of genes belonging to the MAPK signaling pathway, focal adhesion, regulation of the actin cytoskeleton, Rap1 signaling pathway et al. only enriched in comparisons of Group MNP+MNA+ vs. MNP+MNAand MNP+MNA+ vs. MNP-. This indicates that MNA is an independent factor responsible for the differential expression of certain genes (Fig. 5A). To gain a more biological context, we further assigned the DEGs to defined biological function terms by using Gene Ontology databases (Fig. 5B). Consistently, gene sets of cell differentiation, ERK cascade signal transduction (which is downstream of RAS/MAPK), cell adhesion, and cellular response to TGF- $\beta$ stimulus were enriched only in Group $\mathrm{MNP}+\mathrm{MNA}+$ vs. MNP+MNA - and MNP+MNA+ vs. $\mathrm{MNP}-$, which further confirmed the unique alteration of signal transduction and cell-substrate interaction. By dissecting the top enriched gene sets in $\mathrm{GO}$ analysis, we found significant downregulation of Crkl and Gdnf, reported inhibitors of osteogenic differentiation, in Group $\mathrm{MNP}+\mathrm{MNA}+$, and upregulation of Smad8 and Ngf, wellknown genes that promote osteogenic differentiation, as visualized by the heat map (Fig. 5C). To further identify the pathways that contribute to $\mathrm{MNP}+\mathrm{MNA}+$-induced osteogenesis, we selected 201 genes with differential expression only in Group MNP+MNA+ (Fig. 5D). A dot plot map of KEGG pathway analysis revealed that the majority of enrichment was in the MAPK signaling pathway, focal adhesion, regulation of the actin cytoskeleton, and PI3K-AKT signaling pathway. Combined with the results of Fig. 5A, these data implied that the key cellular pathways responded to alterations in the microenvironment generated by MNP $+\mathrm{MNA}+$ towards osteogenic differentiation, which is summarized in detail in Fig. 6.

Increasing the efficiency of directional MCS osteogenic differentiation has long been a central issue in bone tissue engineering. Most recent studies have focused on using exogenous biochemical cues, e.g., growth factors, whose use in bone defect repair has aroused clinical safety concerns ${ }^{2}$. Leveraging physical factors in bone tissue engineering scaffolds to induce MCS osteogenic differentiation opens a new door for addressing this issue. Tailoring the mechanical properties (e.g., Young's modulus) of scaffolds to support MCS osteogenic differentiation was pioneered by A. Engler ${ }^{5}$ and others ${ }^{21}$, and its feasibility has been extensively validated. However, using other physical factors, e.g., anisotropicity, which is also universally present in living tissue and organs, to induce directional MCS osteogenic differentiation, has been relatively less extensively explored and utilized. There are multiple reasons for this fact, and one might be that the specificity of anisotropicity in the induction of MCS osteogenesis is not sufficiently high, although various studies have reported that anisotropicity of the substrate supports MCS osteogenic differentiation ${ }^{15,22,23}$.

With this idea in mind and considering the scenario in real tissues or organs that have anisotropic structures at different scale levels, we proposed a model substrate that has anisotropic structures at dual scale levels. Specifically, a 3D printing technique was used to print PDMS stripes on the hundred-micrometer scale, while a field-induced magnetic nanoparticle assembly technique was used to fabricate anisotropic MNAs on the micrometer scale. PDMS and ferumoxytol were used in this study because of their excellent biocompatibility, and they are widely used in biomedical fields, for example, to establish in vitro models (e.g., PDMS for organ-on-chips ${ }^{24}$ and MNA for multicellular spheroids ${ }^{25}$ ) for testing novel therapeutic interventions. We hypothesize that such anisotropicity at dual-scale levels will increase the ability of ADSCs to induce osteogenic differentiation. Of note, the scale level of anisotropic MNAs approaches the size of a single ADSC. Specifically, the size of single nanoparticles is on the $10-100 \mathrm{~nm}$ scale and that of assemblies is approximately $10-20 \mu \mathrm{m}$ (Fig. 3I). In this context, although the ADSCs are substantially larger than a single assembly, they are on the same order of magnitude, especially considering that the size of cells is relatively variable in different scenarios (fully spread under 2D conditions vs. relatively spherical under 3D/quasi 3D conditions). However, here, the main claim we would like to make is that the scale of these anisotropic structures is in line with that of whole cells, rather than in line with or even smaller than that of subcellular structures (e.g., pseudopodia) or substantially larger than that of whole cells. Examples of the former could include fibrous scaffolds generated from electrospinning, and examples of the latter could include porous tissue engineering scaffolds. We expect the anisotropicity that single ADSCs can sense to be maximized within the microenvironment in this study, while 


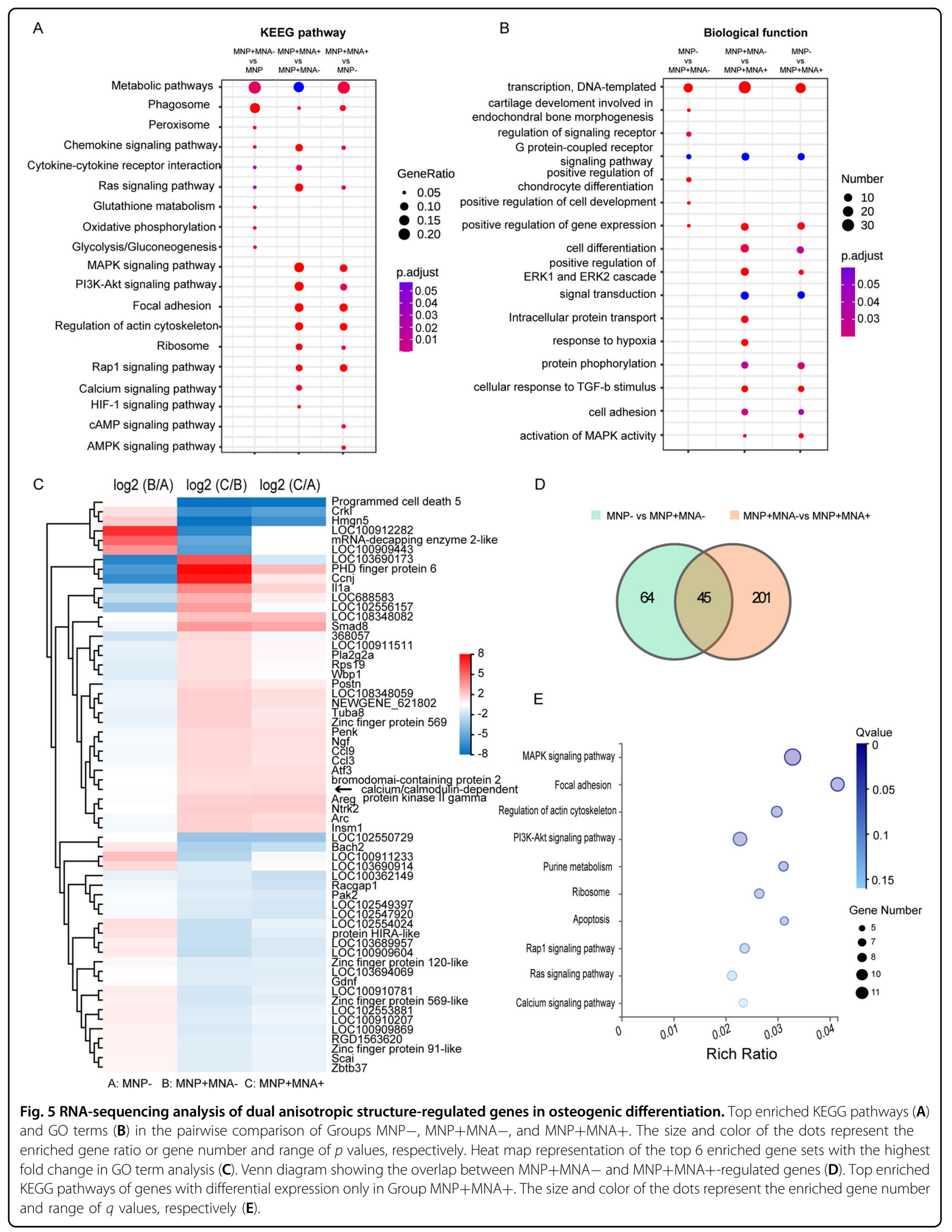




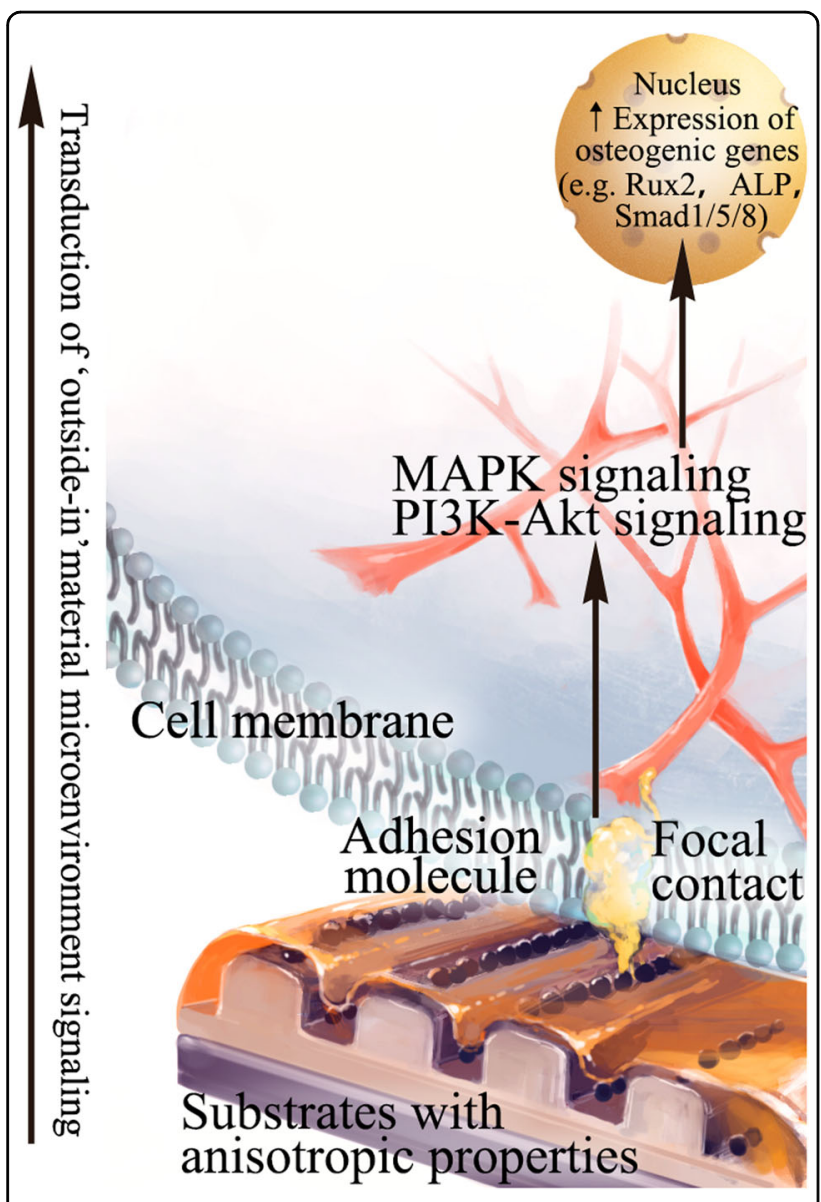

Fig. 6 Model for proposed mechanism of dual anisotropic structures in promoting osteogenic differentiation. While chemical factors promote osteogenic differentiation, dual anisotropic structures, likely upregulted signaling pathways including MAPK signaling and PI3K-Akt signalings to enhance transcriptional-activator function of RUNX2 and SMADs by modulating the interaction between ADSCs and substrate.

anisotropic structures an order of magnitude larger or smaller are still isotropic to single ADSCs. For example, the sizes of those channels or pores that are present in conventional bone tissue engineering are hundreds of microns or even larger. Macroscopically, those scaffolds were still anisotropic; however, within the area where a single ADSC spread over the topography is still isotropic with only minor curvature; we suspect that such topography could not provide any anisotropic clues to a single ADSC.

To validate these hypotheses, we compared the substrates with optimized antistrophic PDMS stripes only or PDMS stripes with magnetic nanoparticles coated in the absence or presence of a magnetic field (Group MNP-, Group MNP+MNA-, and Group MNP+MNA+, respectively) with respect to several osteogenesis markers expressed by ADSCs at the protein and mRNA levels.
Calcium nodules were found to be significantly highly expressed in Group MNP+MNA+, consistent with ALP measurement, which was most strongly expressed in this group at both the protein and mRNA levels and other osteogenesis markers, such as BMP-1 and RUNX2. These sets of data support our hypothesis that such dual-scale (Group MNP+MNA+) anisotropic structures collaboratively increase the ADSC osteogenesis differentiation induction ability of the substrate.

Of note, with these settings, an antistrophic micromagnetic field was introduced into the substrate generated from anisotropic MNA. The FMR spectrum demonstrated that with respect to the substrate with fieldinduced magnetic nanoparticle assemblies, the magnetic properties were stronger parallel to the assemblies than perpendicular to the assemblies. This is consistent with our previous finding ${ }^{17}$, and we speculate that such anisotropic magnetic properties contribute to ADSC osteogenic differentiation as well. In fact, using a magnetic field to control stem cell differentiation has increasingly attracted research interest in recent years. Various studies have shown that magnetic fields or magnetic nanomaterials, alone or in combination with other conventional bone repair materials, could contribute to the osteogenic differentiation of stem cells and promote bone repair ${ }^{26-28}$. Current magnetic nanomaterial-polymer composite fabrication approaches include coating nanomaterials on the surface of polymer substrates and blending magnetic nanomaterials with polymers. The coating approach limits the total amount of magnetic nanomaterials that can be contained in the composite, and the shedding of nanomaterials from the substrate during exposure to mechanical forces is another issue, even if the nanomaterial is covalently bound to the substrate. While the encapsulation of nanomaterials in polymer substrates can partly address this issue, the direct interaction between magnetic materials and cells will be greatly reduced by the isolation of magnetic materials from the cells by the polymer substrate. With these limitations, both of these approaches fail to fully present the unique magnetic and surface interfaces of superparamagnetic nanomaterials. The designed substrate with MNA can at least partly address this issue since compared to single nanoparticles, the assemblies are potentially more resistant to shedding in response to mechanical forces ${ }^{29}$ or endocytosis by cells, while the magnetic properties of the nanomaterials are increased by the field-induced assembly. This can therefore also decrease the amount of MNPs used to fabricate substrates with the same magnetic properties as those with single MNPs and consequently avoid excessive iron ion concentrations via the metabolism of composite materials. With these advantages, we believe that such a substrate can serve as a candidate platform to investigate the influence of magnetic fields that are externally 
generated or generated from MNPs on the osteogenesis of ADSCs or other stem cells in future studies. The following questions are also worth mentioning here, although they are beyond the scope of this article. First, we still take safety issues into consideration, as we expect potential clinical translation of this concept; therefore, ferumoxytol, an FDA-approved iron oxide nanoparticle for iron supplementation $^{30}$, was used in this study as the MNA building block. Second, we expect that the density of cell anchorage points, which is mediated by magnetic nanoassemblies, also plays roles in regulating the osteodifferentiation of ADSCs, which is supported by the RNAseq data showing that focal adhesion was differentially regulated during differentiation (Fig. 5E). This is consistent with our previous finding that the density of anchorage points could significantly mediate cellular signaling ${ }^{31}$. At the current stage, although the role of magnetic properties and anisotropicity in the induction of ADSC osteogenic differentiation should be fully elucidated before considering animal-based validation experiments, it is still suitable in vitro platform for mechanistic studies and therapeutic candidate testing. In addition, we believe that combination with cell-derived ECM will improve the cell-composite interaction and further promote the osteogenesis of stem cells ${ }^{32-34}$.

\section{Conclusion}

In conclusion, we designed a substrate with a dual anisotropic structure fabricated via a combination of 3D printing and field-induced magnetic nanoparticle assemblies. These dual anisotropic structures have a scale hierarchy, and the scale of the magnetic nanoparticle assemblies matches that of a single ADSC. Alizarin red staining, q-PCR, western blot, and RNA-seq data consistently demonstrated that ADSCs cultured on substrates with these structures have a stronger osteogenesis tendency. In particular, RNA-seq data revealed that the genes that mainly responded to dual anisotropic structures were enriched in cell adhesion, cytoskeleton, and kinase signaling pathways, including MAPK pathways and PI3KAkt pathways. These data support our hypothesis that such a structure could maximize the microenvironmental anisotropicity sensed by ADSCs and therefore validate the feasibility of this strategy in promoting ADSC osteogenic differentiation. This not only sheds light on the mechanism by which anisotropic structures induce MCS osteogenic differentiation but also provides new insight into the design of bone tissue engineering scaffolds.

\section{Materials and methods Materials}

OriCell Sprague Dawley Rat Adipose-derived Stem Cell (SD rADSCs), SD Rat Adipose-derived Stem Cell Osteogenic Differentiation Medium, and SD Rat Adipose-derived
Mesenchymal Stem Cell Complete Medium were purchased from Cyagen Biosciences (Guangzhou, China). Other reagents used in this study include $0.25 \%$ TrypsinEDTA (Thermo Fisher Scientific, Waltham, MA, USA), RIPA (Beyotime Biotech., Shanghai, China), RNAiso Plus (9108Q; Takara Bio Inc.; Japan), Prime Script ${ }^{\mathrm{TM}}$ RT Master Mix (RR036A; Takara Bio Inc.), TB Grenn ${ }^{\mathrm{TM}}$ Premix Ex $\mathrm{Taq}^{\mathrm{TM}}$ II (RR820A; Takara Bio Inc.), Cell Counting Kit-8 (CCK-8; E1CK-000208; Enogene Biotech., Nanjing, China), rhodamine-phalloidin (Cytoskeleton, Inc., Denver, CO, USA), 4;,6-diamidino-2-phenylindole (DAPI; Beyotime Biotech.), alkaline phosphatase (ALP) assay kit (A059-2-2; Jiancheng Bioengineering Institute, Nanjing, China), bicinchoninic acid protein assay kit (BCA; PT001; Leagene Biotech., Beijing, China), cetylpyridinium chloride (Bomei Biotechnology, Hefei, China), alizarin red S (ARS; Cyagen Biosciences Inc., Guangzhou, China), antibodies (Abcam, USA), and chemiluminescence reagent (NOWBKCS0100; Millipore, USA).

\section{Preparation of the magnetic composite scaffold}

The magnetic composite scaffolds used for cell culture were fabricated via 3D printing and magnetic fieldassisted self-assembly. The glass slides used as substrates for 3D printing $(10 \mathrm{~mm} \times 10 \mathrm{~mm} \times 1 \mathrm{~mm})$ were ultrasonically cleaned for $30 \mathrm{~min}$ and dried in an electrothermostatic blast oven. After drying, clean glass slides were obtained and ready for use. PDMS (DOW CORNING, SE 1700 CLEAN BASE) and curing agent (DOW CORNING, SE 1700 CATALYST) were weighed and combined at a mass ratio of 9:1, and the mixture was homogenized by mechanical agitation to obtain the PDMS for use. A certain amount of the prepared PDMS was completely dissolved with diethyl ether to obtain a clear solution. A thin layer of PDMS was formed on the surface of the precleaned glass slides by low-speed spin coating ( $<500$ r.p.m.) with PDMS solution. After the ether was evaporated, the slides were placed in an electrothermostatic blast oven to cure the PDMS. An appropriate amount of the prepared PDMS was filled in the material tube of the 3D printer (Adventure Technology, Adventure 3D-LB-Printer), and a print nozzle of a specific size was installed. The scaffold was printed according to the set printing parameters, and the printed scaffold was placed in a dry box to be cured (overnight). After curing, the surface of the scaffold was treated with plasma for $30 \mathrm{~s}$ (air atmosphere). Five milliliters of alkaline dopamine solution ( $1 \mathrm{mg} / \mathrm{mL}, \mathrm{pH}: 8.5)$ was prepared, and the PDMS scaffold was immersed in the solution for $12 \mathrm{~h}$ at $4{ }^{\circ} \mathrm{C}$. After $12 \mathrm{~h}$, the alkaline dopamine solution was discarded, and the PDMS scaffold was washed with pure water three times. The magnetic nanomaterial used in our work was the only inorganic nanodrug approved by the FDA, ferumoxytol $\left(\gamma-\mathrm{Fe}_{2} \mathrm{O}_{3} @ P S C\right)$, which was kindly provided by 
Jiangsu Key Laboratory for Biomaterials and Devices. Then, $100 \mu \mathrm{L}$ of $\gamma-\mathrm{Fe}_{2} \mathrm{O}_{3} @ P S C$ solution $(0.5 \mathrm{mg} / \mathrm{mL})$ was added to the surface of the 3D-printed scaffold in a nonmagnetic field, while $100 \mu \mathrm{L}$ of a $\gamma-\mathrm{Fe}_{2} \mathrm{O}_{3} @ \mathrm{PSC}$ $(0.5 \mathrm{mg} / \mathrm{mL})$ and polylysine $\left(\gamma-\mathrm{Fe}_{2} \mathrm{O}_{3} @ \mathrm{PSC}\right.$ : polylysine $=$ 1:3.5) mixture was added to a magnetic field. The assembled structure of the magnetic nanomaterial was formed and fixed on the surface of the scaffold via solvent evaporation. A total of $120 \mu \mathrm{L}$ of dopamine solution $(1 \mathrm{mg} / \mathrm{mL})$ and collagen type I (CORNING, Rat Tail, $0.5 \mathrm{mg} / \mathrm{mL}$ ) mixture was prepared and added to the surface of the magnetic composite scaffold.

\section{Scanning/transmission electron microscope characterization}

SEM images of the magnetic composite scaffold were obtained with a LEO1530VP system (Zeiss Germany). TEM images of $\gamma-\mathrm{Fe}_{2} \mathrm{O}_{3} @ P S C$ were obtained with a JEM2000EX system (JEO Japan).

\section{Ferromagnetic resonance characterization}

Since the size of the three-dimensional printing scaffold for cell culture $(20 \times 20 \times 1 \mathrm{~mm})$ is too large to be used as a sample for ferromagnetic resonance (FMR) detection, a three-dimensional printing scaffold with reduced size $(5 \times$ $5 \times 1 \mathrm{~mm}$ ) but the same preparation procedures were used for FMR measurement.

The magnetic properties of the sample containing the assembled structure and the assembly-free structure were separately detected by FMR. Each sample was detected in two directions: with the long axis of the printed structure parallel to the direction of the FMR magnetic field and with the long axis perpendicular to the direction of the magnetic field. Finally, their respective FMR spectral curves were obtained and summarized in the same coordinate system for comparison. The samples were found to have magnetic anisotropy.

\section{Cell seeding}

Before cell seeding, the PDMS was first washed with PBS $(\mathrm{pH}=7.4)$ twice and then conditioned in SD rat adipose-derived mesenchymal stem cell complete medium (RASMD-90011; Cyagen Biosciences Inc., Guangzhou, China) for $24 \mathrm{~h}$. SD rADSCs were cultured in SD rADSC complete medium at $37^{\circ} \mathrm{C}$ in a humidified incubator in the presence of $5 \% \mathrm{CO}_{2}$. Cells at passages 3-5 were used in our experiments. When confluence reached approximately $80 \%$, the cells were trypsinized and resuspended at a concentration of $3 \times 10^{6} \mathrm{cell} / \mathrm{mL}$. To allow cells to adhere only to the surface of PDMS rather than everywhere in the well plate, $60 \mu \mathrm{L}$ of cell suspension was added on top of each PDMS $\left(1.8 \times 10^{5}\right.$ cells/well), followed by the addition of $2 \mathrm{~mL}$ of the complete medium after incubation for $4 \mathrm{~h}$. The culture medium was changed every 2 days. When confluence reached approximately $80 \%, 2 \mathrm{~mL}$ SD rat adipose-derived stem cell osteogenic differentiation medium was added to each well. The medium was changed every 3 days. The morphology of SD rADSCs adhering to the scaffolds was viewed using an inverted microscope (LEICA DMC2900; Carl Zeiss Meditec AG, Germany) on a bright field and then photographed at $\times 400$ magnification using LAS Vision 4.4 software.

\section{Confocal laser scanning microscopy (CLSM) characterization}

Cells were seeded using the above method. The cells were fixed using $4 \%$ polyformaldehyde for $30 \mathrm{~min}$ and permeabilized using $0.5 \%$ Triton X-100 (Solarbio Life Science) for $30 \mathrm{~min}$. Then, the cells were stained with rhodamine-phalloidin (Cytoskeleton, Inc., Denver, CO, USA) and 4',6-diamidino-2-phenylindole (DAPI; Beyotime Biotechnology) to label F-actin and cell nuclei, respectively. The morphology of rADSCs adhering to PDMS was observed by CLSM (Zeiss-LSM710; Carl Zeiss Meditec AG, Jena, Germany).

\section{ALP activity assay}

The SD rADSCs were seeded using the above method. After incubation in osteogenic medium for 4 and 7 days, SD rADSCs were collected using $0.5 \%$ trypsin for analysis. ALP activity and total protein concentration were detected according to the manufacturer's instructions for the ALP assay kit (A059-2-2; Jiancheng Biotech., Nanjing, China) and bicinchoninic acid protein assay kit (PT001; Leagene Biotech., Beijing, China), respectively. The ALP concentration was calculated according to the manufacturer's instructions.

\section{Matrix mineralization assay}

Matrix mineralization levels were evaluated by Alizarin red staining. After osteogenic induction for 14 days, rADSCs on the samples were fixed with $4 \%$ paraformaldehyde and stained with 1\% ARS ( $\mathrm{pH} 4.2$; Cyagen Biosciences Inc., Guangzhou, China) for $5 \mathrm{~min}$. After qualitative observation, the samples were washed with PBS and extracted with $10 \%$ cetylpyridinium chloride (Bomei Biotechnology, Hefei, China) for $30 \mathrm{~min}$ at room temperature. Then, the solution was transferred to a 96well plate for absorbance measurement at $550 \mathrm{~nm}$ using a microplate reader.

\section{Quantitative RT-PCR}

After incubation in osteogenic medium for 14 days, SD rADSCs were collected using $0.5 \%$ trypsin for analysis. Total RNA was extracted using RNAiso Plus (Takara Bio Inc.; Takara Bio Inc., Japan). The quantity and quality of RNA were measured with NanoVue Plus (General Electric 
Company, USA). cDNA was synthesized from $500 \mathrm{ng}$ of RNA using Prime Script ${ }^{\mathrm{TM}}$ RT master Mix (RR036A; Takara Bio Inc., Japan) according to the manufacturer's instructions. Quantitative real-time PCR was performed using the ABI 7900 Real-Time PCR System (Thermo Fisher Scientific) with TB Grenn ${ }^{\mathrm{TM}}$ Premix Ex $\mathrm{Taq}^{\mathrm{TM}}$ II (RR820A; Takara Bio Inc., Japan) according to the manufacturer's instructions. The analysis was performed using the $2-\Delta \Delta \mathrm{Ct}$ method. Each experiment was run in triplicate. The primer sequences of OCN (osteocalcin), BMP2 (bone morphogenetic protein 2), OSX (osterix), Runx-2 (runt-related transcription factor 2), ALP (alkaline phosphatase), and Smad1/5/8 (Sma-Mad) are listed in Table S1.

\section{Western blot analysis}

Western blotting was performed as previously described. Immunoblotting was performed using appropriate primary antibodies against ALP, BMP2, RUN2, Smad1/5/ 8, and GAPDH (ImageQuant LAS 4000 mini).

\section{RNA sequencing and data analysis}

RNA from MNP-, MNP $+\mathrm{MNA}-$, and MNP $+\mathrm{MNA}+$ cultured rat adipose-derived stem cells was extracted using TRIzol (Life Technologies), followed by purification using an RNeasy Mini Kit (Qiagen). RNA-seq was performed using rat adipose-derived stem cells from three individual animals. RNA-seq libraries were prepared using the Illumina TruSeq RNA Library Prep Kit v2 and sequenced with a HiSeq 2500 sequencer.

\section{Statistical analysis}

Each type of experiment was performed at least three times independently, and data are expressed as the mean \pm SD. Statistical analysis among different groups was carried out by Student's t-test. $P$ values $<0.05$ were considered to be statistically significant. Statistical analyses were performed using GraphPad Prism software (version 5.0).

\section{Acknowledgements}

This work was supported by grants from the National Key Research and Development Project (2016YFA0201704), the National Natural Science Foundation of China (81870807, 81701824, and 51832001), the China Postdoctoral Science Foundation (2017M621787), the Talent Introduction Foundation of Nanjing Medical University (2017RC07) and the Priority Academic Program Development of Jiangsu Higher Education Institutions (PAPD, 2018-87)

\footnotetext{
Author details

'Jiangsu Key Laboratory of Oral Diseases, Affiliated Hospital of Stomatology, Nanjing Medical University, Nanjing, Jiangsu, China. ${ }^{2}$ Laboratory of Oral Regenerative Medicine Technology, School of Biomedical Engineering and Informatics, Department of Biomedical Engineering, Nanjing Medical University, Nanjing, Jiangsu, China. ${ }^{3}$ Department of Medical Genetics, School of Basic Medical Science and Jiangsu Key Laboratory of Xenotransplantation, Nanjing Medical University, Nanjing, Jiangsu, China. ${ }^{4}$ Institute for Nanobiotechnology, Johns Hopkins University, Baltimore, MD 21218, USA.
}

${ }^{5}$ Jiangsu Key Laboratory for Biomaterials and Devices, School of Biological Science and Medical Engineering, Southeast University, Nanjing, Jiangsu, China. ${ }^{6}$ Materials Science and Devices Institute, Suzhou University of Science and Technology, 1 Kerui Road, Suzhou, Jiangsu, China

\section{Conflict of interest}

The authors declare no competing interests.

\section{Publisher's note}

Springer Nature remains neutral with regard to jurisdictional claims in published maps and institutional affiliations.

Supplementary information The online version contains supplementary material available at https://doi.org/10.1038/s41427-021-00288-x.

Received: 14 September 2020 Revised: 7 January 2021 Accepted: 25 January 2021.

Published online: 5 March 2021

\section{References}

1. Amini, A. R., Laurencin, C. T. \& Nukavarapu, S. P. Bone tissue engineering: recent advances and challenges. Crit. Rev. Biomed. Eng. 40, 363-408 (2012).

2. Toosi, S. \& Behravan, J. Osteogenesis and bone remodeling: a focus on growth factors and bioactive peptides. BioFactors 46, 326-340 (2020).

3. Guilak, F. et al. Control of stem cell fate by physical interactions with the extracellular matrix. Cell Stem Cell 5, 17-26 (2009).

4. Higuchi, A., Ling, Q.-D., Chang, Y., Hsu, S.-T. \& Umezawa, A. Physical cues of biomaterials guide stem cell differentiation fate. Chem. Rev. 113, 3297-3328 (2013).

5. Engler, A. J., Sen, S., Sweeney, H. L. \& Discher, D. E. Matrix elasticity directs stem cell lineage specification. Cell 126, 677-689 (2006).

6. Chaudhuri, $\mathrm{O}$. et al. Hydrogels with tunable stress relaxation regulate stem cell fate and activity. Nat. Mater. 15, 326-334 (2016)

7. LaPointe, V. L., Fernandes, A. T., Bell, N. C., Stellacci, F. \& Stevens, M. M. Nanoscale topography and chemistry affect embryonic stem cell self-renewal and early differentiation. Adv. Healthc. Mater. 2, 1644-1650 (2013).

8. Cockerill, I. et al. Micro-/nano-topography on bioresorbable zinc dictates cytocompatibility, bone cell differentiation, and macrophage polarization. Nano Lett. 20, 4594-4602 (2020).

9. Metavarayuth, K., Sitasuwan, P., Zhao, X., Lin, Y. \& Wang, Q. Influence of surface topographical cues on the differentiation of mesenchymal stem cells in vitro. ACS Biomater. Sci. Eng. 2, 142-151 (2016).

10. Tabor, Z., Petryniak, R., Latała, Z. \& Konopka, T. The potential of multi-slice computed tomography based quantification of the structural anisotropy of vertebral trabecular bone. Med. Eng. Phys. 35, 7-15 (2013).

11. Park, D. et al. Extracellular matrix anisotropy is determined by TFAP2Cdependent regulation of cell collisions. Nat. Mater. 19, 227-238 (2020).

12. Wong, S. H. D. et al. Anisotropic nanoscale presentation of cell adhesion ligand enhances the recruitment of diverse integrins in adhesion structures and mechanosensing-dependent differentiation of stem cells. Adv. Funct. Mater. 29, 1806822 (2019).

13. $\mathrm{Yu}, \mathrm{Y}$. et al. Regulation of osteogenesis by micro/nano hierarchical titanium surfaces through a Rock-Wnt5a feedback loop. Colloids Surf. B 170, 1-10 (2018).

14. Dewey, M. J., Nosatov, A. V., Subedi, K. \& Harley, B. Anisotropic mineralized collagen scaffolds accelerate osteogenic response in a glycosaminoglycandependent fashion. RSC Adv. 10, 15629-15641 (2020).

15. Azeem, A. et al. The influence of anisotropic nano-to micro-topography on in vitro and in vivo osteogenesis. Nanomedicine 10, 693-711 (2015).

16. Rho, J.Y., Kuhn-Spearing, L. \& Zioupos, P. Mechanical properties and the hierarchical structure of bone. Med. Eng. Phys. 20, 92-102 (1998).

17. $\mathrm{Hu}, \mathrm{K}$. et al. A novel magnetic hydrogel with aligned magnetic colloidal assemblies showing controllable enhancement of magnetothermal effect in the presence of alternating magnetic field. Adv. Mater. 27, 2507-2514 (2015).

18. $\mathrm{Hu}, \mathrm{K}$. et al. Sliced magnetic polyacrylamide hydrogel with cell-adhesive microarray interface: a novel multicellular spheroid culturing platform. ACS Appl. Mater. Interfaces 8, 15113-15119 (2016). 
19. Tang, S. et al. High quality multicellular tumor spheroid induction platform based on anisotropic magnetic hydrogel. ACS Appl. Mater. Interfaces 9 10446-10452 (2017)

20. Guo, Z. et al. Fabrication of hydrogel with cell adhesive micropatterns for mimicking the oriented tumor-associated extracellular matrix. ACS Appl. Mater. Interfaces 6, 10963-10968 (2014).

21. Shih, Y. R. V., Tseng, K. F., Lai, H. Y., Lin, C. H. \& Lee, O. K. Matrix stiffness regulation of integrin-mediated mechanotransduction during osteogenic differentiation of human mesenchymal stem cells. J. Bone Miner. Res. 26, 730-738 (2011).

22. Yang, L., Ge, L., Zhou, Q., Jurczak, K. \& van Rijn, P. Decoupling amplitude and wavelength of anisotropic topography and the influence on osteogenic differentiation of mesenchymal stem cells using a high-throughput screening approach. ACS Appl. Bio Mater 3, 3690-3697 (2020).

23. Peng, R., Yao, X. \& Ding, J. Effect of cell anisotropy on differentiation of stem cells on micropatterned surfaces through the controlled single cell adhesion. Biomaterials 32, 8048-8057 (2011).

24. Guo, Z. et al. Validation of a vasculogenesis microfluidic model for radiobiological studies of the human microvasculature. Adv. Mater. Technol. 4, 1800726 (2019)

25. Yu, T., Hu, Y., Feng, G., Hu, K. \& Graphene-Based, A. Flexible device as a specific far-infrared emitter for noninvasive tumor therapy. Adv. Therapeutics 3, 1900195 (2020).

26. Wang, Q. et al. Magnetic iron oxide nanoparticles accelerate osteogenic differentiation of mesenchymal stem cells via modulation of long noncoding RNA INZEB2. Nano Res. 10, 626-642 (2017).
27. Henstock, J. R., Rotherham, M., Rashidi, H., Shakesheff, K. M. \& El Haj, A. J. Remotely activated mechanotransduction via magnetic nanoparticles promotes mineralization synergistically with bone morphogenetic protein 2: applications for injectable cell therapy. Stem Cells Transl. Med. 3, 1363-1374 (2014).

28. Yun, H.-M. et al. Magnetic nanocomposite scaffolds combined with static magnetic field in the stimulation of osteoblastic differentiation and bone formation. Biomaterials 85, 88-98 (2016).

29. Sun, J. et al. Magnetic assembly-mediated enhancement of differentiation of mouse bone marrow cells cultured on magnetic colloidal assemblies. Sci. Rep. 4, 5125 (2014).

30. Lu, M., Cohen, M. H., Rieves, D. \& Pazdur, R. FDA report: ferumoxytol for intravenous iron therapy in adult patients with chronic kidney disease. Am. J. Hematol. 85, 315-319 (2010).

31. $\mathrm{Yu}, \mathrm{T}$. et al. An easy-to-fabricate hydrogel platform with tunable stiffness and cell anchorage: validation of its feasibility in modulating sonic hedgehog signaling pathway physically. Macromol. Mater. Eng. 305, 1900759 (2020)

32. Yang, L., Ge, L. \& V. Rijn, P. Synergistic effect of cell-derived extracellular matrices and topography on osteogenesis of mesenchymal stem cells. ACS Appl. Mater. Interfaces 12, 25591-25603 (2020).

33. Yang, L., Jurczak, K. M., Ge, L. \& v. Rijn, P. High-throughput screening and hierarchical topography-mediated neural differentiation of mesenchymal stem cells. Adv. Healthc. Mater. 9, 2000117 (2020).

34. Yang, L. et al. Topography induced stiffness alteration of stem cells influences osteogenic differentiation. Biomater. Sci. 8, 2638-2652 (2020). 